\title{
Searching Hubble Space Telescope Images for Core-Collapse Supernova Progenitors
}

\author{
Schuyler D. Van Dyk \\ IPAC/Caltech, 100-22, Pasadena, CA 91125, USA \\ Weidong Li \& Alexei V. Filippenko \\ Astronomy Department, University of California, Berkeley, CA 94720, \\ USA
}

\begin{abstract}
Identifying the massive progenitor stars that give rise to core-collapse supernovae $(\mathrm{SNe})$ is one of the main pursuits of supernova and stellar evolution studies, and is essential for understanding the birth of pulsars. Using ground-based images of recent, nearby SNe obtained primarily with the Katzman Automatic Imaging Telescope (KAIT), astrometry from 2MASS, and archival images from the Hubble Space Telescope $(H S T)$, we have attempted the direct identification of the progenitors of 16 Type II and Type Ib/c SNe. We may have identified the progenitors of the Type II SNe 1999br and 1999ev, the Type Ib SNe 2001B and 2001is, and the Type Ic SN 1999bu, possibly doubling the number of known SN progenitors. For the remaining SNe, limits placed on the absolute magnitude and color (when available) of the progenitor allows us to place limits on the progenitor's mass. Specifically, we have been able to place a relatively stringent limit on the progenitor of the Type II-P SN 2001du in NGC 1365, consistent with the limits placed on the masses of other Type II-P SNe. We have also recently identified the progenitor of the Type II-P SN 2003gd in Messier 74.
\end{abstract}

\section{Introduction}

Before young neutron stars, there must be supernovae ( $\mathrm{SNe}$ ), and before $\mathrm{SNe}$, there must be supernova (SN) progenitor stars. The SNe responsible for neutron stars are thought to be core-collapse events, which occur for stars with initial mass $M_{\text {ZAMS }} \precsim 8-10 \mathrm{M}_{\odot}$ (e.g., Woosley \& Weaver 1986). The range of possible masses for core-collapse SN progenitors must somehow result in the range of observed masses for young neutron stars (e.g., Thorsett \& Chakrabarty 1999). Identifying the massive progenitor stars which give rise to core-collapse $\mathrm{SNe}$ is one of the main pursuits of supernova and stellar evolution studies.

Core-collapse SNe come in various types: (1) the Type II-plateau (II-P), which exhibit a sustained plateau in the multi-band optical light curves for $\sim 100$ days after maximum, coupled with classical broad-width $\mathrm{P}$ Cygni Balmer line profiles in their optical spectra (e.g., SN 1999em); (2) the Type II-linear (IIL), which lack the plateau, but instead show a steady decline after maximum, 
followed by an exponential tail powered by radioactive ${ }^{56} \mathrm{Ni}$ decay, as well as weak or no blue-shifted absorption troughs in the Balmer profiles (e.g., SN 1979C); (3) the Type II-narrow (IIn), which show a relatively narrow emission profile atop a broad base in the Balmer lines, and, in the more extreme cases, a featureless blue continuum near maximum (e.g., SN 1988Z); the Type Ib, which lack H, and also the Si II absorption feature which characterizes Type Ia SNe, but instead show prominent He lines (e.g., SN 1983N); the Type Ic, which resemble the Type Ib, but show weak or no He (e.g., SN 1994I); and, the Type IIb, which, for the first month or so, resemble SNe II-P, but subsequently transform to resemble $\mathrm{SNe} \mathrm{Ib}$ (with broad Balmer profiles, again, at late times; e.g., SN 1993J). See Filippenko (1997) for a thorough review of SN spectra and types.

The main obstacle to identifying the progenitor is that a SN leaves few traces of the star that exploded. Before now, only five out of (at the time of this writing) nearly 2900 historical extragalactic SNe have had their progenitors directly identified. These include SN $1961 \mathrm{~V}$ in NGC 1058 (Zwicky 1964, 1965), SN 1978K in NGC 1313 (Ryder et al. 1993), SN 1987A in the LMC (e.g., Gilmozzi et al. 1987; Sonneborn, Altner \& Kirshner 1987), SN 1993J in M81 (Aldering, Humphreys \& Richmond 1994; Cohen, Darling \& Porter 1995), and SN 1997bs in M66 (Van Dyk et al. 1999). It should be noted that these five SNe were all at least somewhat unusual, and both SNe 1961V (Van Dyk, Filippenko \& Li 2002) and 1997bs (Van Dyk et al. 2000) may not have been actual SNe.

\section{SN Progenitor Search}

Clearly, direct identification of the progenitors of additional core-collapse $\mathrm{SNe}$ is essential. We have exploited the superior spatial resolution afforded by HST to isolate the progenitors of six SNe II and ten $\mathrm{SNe} \mathrm{Ib} / \mathrm{c}$ in WFPC2 images (Van Dyk, Li \& Filippenko 2003a). The crux is determining the SN location on one of the four WFPC2 chips. It is therefore of utmost importance to have high astrometric accuracy for all image data. Ideally, one could pinpoint the exact SN location by comparing a late-time SN image with a pre-SN image. However, this option was usually not available, and we had to measure the SN position on a KAIT image and then locate the SN site by applying an independent astrometric grid to the pre-SN image. We adopted 2MASS (with positional uncertainty $\left.\lesssim 0^{\prime \prime} 10\right)$ as the basis for the grid for both the ground-based and HST images. Once the SN site was located, photometry of the appropriate WFPC2 chip was performed using the routine HSTphot (Dolphin 2000a,b) with a 3- $\sigma$ detection threshold.

We had possibly identified the progenitors of SNe II $1999 \mathrm{br}, 1999 \mathrm{ev}$, and $2001 \mathrm{du}$ as supergiant stars with $M_{V}^{0} \approx-6 \mathrm{mag}$, and the progenitors of $\mathrm{SNe} \mathrm{Ib}$ $2001 \mathrm{~B}$ and 2001 is as very luminous supergiants with $M_{V}^{0} \approx-8$ to $-9 \mathrm{mag}$, as well as the progenitor of SN Ic 1999bu as a supergiant with $M_{V}^{0} \approx-7.5 \mathrm{mag}$. For all other $\mathrm{SNe}$ in our sample we could only place limits on the progenitor absolute magnitude and color. We also recovered SNe $1999 \mathrm{dn}, 2000 \mathrm{C}$, and $2000 \mathrm{ew}$ at late times, but, unfortunately, their pre-SN images did not show a progenitor candidate at the SN position.

Program GO-9353 has imaged six of the SNe at late times in multiple bands with ACS. We had already recovered SN 2000ew, and, along with SNe 1999an 
and 2000ds, we did not detect a SN progenitor in each pre-SN image. We did not correctly identify the progenitor for SN 2001B. For SNe $1999 \mathrm{br}$ and $1999 \mathrm{ev}$, the new observations could still be quite revealing.

\section{The Type II-P SN 2001du in NGC 1365}

For SN 2001du we isolated three possible candidate progenitor stars within the uncertainty of the measured SN position: one reddish star with a detection in both the F555W and F814W bands, and two stars with only F555W detections, implying bluer colors for these latter two stars (Van Dyk et al. 2003a). Late-time multi-band WFPC2 SN images were obtained by program GO-9041. We had assumed that, since SN 2001du is of Type II-P, the most plausible candidate is the redder star. We used the late-time $\mathrm{SN}$ images, specifically the $V$ image, and found the SN position on the pre-SN images to be $0.70 \pm 0.15 \mathrm{WF}$ pixels $\left(0^{\prime} .07\right)$ northeast of one of the blue stars (Van Dyk, Li \& Filippenko 2003b). Thus, we concluded that the progenitor is not detected in the pre-SN images.

We used the F555W and F814W pre-SN image detection limits to constrain the nature of the progenitor: Adopting $E(B-V) \approx 0.1 \mathrm{mag}$ and distance modulus $\mu=31.3 \mathrm{mag}$ for SN $2001 \mathrm{du}$, we infer $M_{V}>-6.4$ and $M_{I}>-7.0 \mathrm{mag}$ for the progenitor star. These limits can be converted to the likely supergiant progenitor luminosity, assuming the full range of possible stellar surface temperatures. Stars with luminosities brighter than these limits should have been detected in the pre-SN images. We compared the limits to model stellar evolutionary tracks for a metallicity appropriate for the SN environment and for a range of masses, and estimated that the $\mathrm{SN}$ progenitor mass is $M_{\mathrm{ZAMS}}<13_{-4}^{+7} \mathrm{M}_{\odot}$.

\section{The Type II-P SN 2003gd in Messier 74 (NGC 628)}

Program GO-9676 had obtained several pre-SN archival WFPC2 images containing the SN site. Using a precise SN position from ground-based images, we isolated the $\mathrm{SN}$ position on the pre-SN images to $\pm 0^{\prime \prime} 6$ ( $\pm 6 \mathrm{WF}$ pixels). Two stars, A and B, were detected near or within the error circle. Color information for both progenitor candidates were obtained from a high-quality, ground-based $I$-band image, on which two faint objects are seen near the positions of both A and B. Both stars are red supergiants, and from model evolutionary tracks for above-solar metallicity, assuming $E(B-V)=0.13 \mathrm{mag}$ and $\mu=29.3 \mathrm{mag}$ for SN 2003gd, Star B had initial mass $M_{\mathrm{ZAMS}} \approx 5 \mathrm{M}_{\odot}$ (formally below the theoretical lower limit for core-collapse $\mathrm{SNe}$ ), and Star A had $M_{\mathrm{ZAMS}} \approx 8-9 \mathrm{M}_{\odot}$. Although Star A is farther from the SN position than Star B, and just outside the edge of the error circle, Star A is the most likely progenitor candidate, based on its initial mass. It is also the most plausible candidate, being the brightest $I$-band object within the SN's larger, $\sim 1^{\prime \prime}$ radius, environment (Van Dyk, Li \& Filippenko 2003c).

\section{Conclusions}

The possible detections and constraints on the SN II progenitors are broadly consistent with red supergiants as progenitor stars, with their colors implying 
spectral types typically earlier than $\mathrm{M}$. The $\mathrm{SN}$ Ib progenitor candidates may be Wolf-Rayet stars, possibly at the upper luminosity end for these stars. The SN Ic progenitor candidate is consistent with a luminous supergiant star. In general, we cannot place rigorous constraints on either the Wolf-Rayet star or massive interacting binary models for $\mathrm{SN} \mathrm{Ib/c} \mathrm{progenitors.}$

The SN 2003gd progenitor is only the sixth ever directly identified. The estimate for its mass is lower than the limits for SNe II-P 1999gi $\left(<15_{-3}^{+5} \mathrm{M}_{\odot}\right.$; Leonard et al. 2002) and 1999em ( $<20 \pm 5 \mathrm{M}_{\odot}$; Leonard et al. 2003), but is consistent with that for SN 2001du. The results suggest that SNe II-P, the most common SNe II and likely the most common origin for young neutron stars, arise from massive stars at the lower extreme of the possible mass range for core collapse. Our search continues ...

Acknowledgments. The work of A.V.F.'s group at UC Berkeley is supported by NSF grant AST-0307894, as well as by NASA grants AR-9953, AR9529, and AR-8754 from the Space Telescope Science Institute, which is operated by AURA, Inc., under NASA contract NAS5-26555. KAIT was made possible by generous donations from Sun Microsystems, Inc., the Hewlett-Packard Company, AutoScope Corporation, Lick Observatory, the National Science Foundation, the University of California, and the Sylvia and Jim Katzman Foundation.

\section{References}

Aldering, G., Humphreys, R. M., \& Richmond, M. W. 1994, AJ, 107, 662

Cohen, J. G., Darling, J., \& Porter, A. 1995, AJ, 110, 308

Dolphin, A. E. 2000a, PASP, 112, 1383

Dolphin, A. E. 2000b, PASP, 112, 1397

Filippenko, A. V. 1997, ARA\&A, 35, 309

Gilmozzi, R., et al. 1987, Nature, 328, 318

Leonard, D. C., et al. 2002, AJ, 124, 2490

Leonard, D. C., et al. 2003, ApJ, 594, 247

Ryder, S., et al. 1993, ApJ, 416, 167

Sonneborn, G., Altner, B., \& Kirshner, R. P. 1987, ApJ, 323, L35

Thorsett, S. E., \& Chakrabarty, D. 1999, ApJ, 512, 288

Van Dyk, S. D., Peng, C. Y., Barth, A. J., \& Filippenko, A. V. 1999, AJ, 118, 2331

Van Dyk, S. D., et al. 2000, PASP, 112, 1532

Van Dyk, S. D., Filippenko, A. V., \& Li, W. 2002, PASP, 114, 700

Van Dyk, S. D., Li, W., \& Filippenko, A. V. 2003a, PASP, 115, 1

Van Dyk, S. D., Li, W., \& Filippenko, A. V. 2003b, PASP, 115, 448

Van Dyk, S. D., Li, W., \& Filippenko, A. V. 2003c, PASP, 115, 1289

Woosley, S. E., \& Weaver, T. A. 1986, ARA\&A, 24, 205

Zwicky, F. 1964, ApJ, 139, 514

Zwicky, F. 1965, in Stars and Stellar Systems, Vol. 8, Stellar Structure, eds. L.

H. Aller, \& D. B. McLaughlin, (Chicago: Univ. Chicago Press), p. 367 